\title{
Artigo/Article
}

\section{Incapacidade pós-tratamento em pacientes hansenianos em Várzea Grande, Estado de Mato Grosso}

\author{
Disability after treatment among leprosy patients in Várzea Grande, State of Mato Grosso
}

José María Hernández Ramos ${ }^{1}$ e Francisco José Dutra Souto²

\begin{abstract}
RESUMO
Introdução: A hanseníase é um importante problema de saúde pública. O Brasil responde por mais de $90 \%$ dos casos do continente americano. Aproximadamente, $23 \%$ dos pacientes hansenianos apresentam algum tipo de incapacidade após a alta. Métodos: Neste estudo, estimou-se a prevalência das incapacidades em 169 pacientes (94 homens e 75 mulheres) com qualquer das formas clínicas de hanseníase no município de Várzea Grande, MT, que já haviam concluído tratamento nos anos anteriores. Foi realizada entrevista e exame clínico da sensibilidade em mãos e pés mediante os filamentos de Semmes-Weinstein. Resultados: A prevalência de incapacidades na amostra analisada foi de $42,6 \%$ sendo $10,1 \%$ de grau dois. Encontrou-se relação estatisticamente significante entre maior prevalência de incapacidades e formas multibacilares de hanseníase. Desde o término do tratamento, 84 (50,2\%) pacientes apresentaram piora evolutiva da sensibilidade, sendo esta piora associada à presença de lesão neural no momento do diagnóstico. Conclusões: Estes resultados enfatizam a importância de monitorar a função neural dos pacientes, muito além do momento de finalizar a poliquimioterapia, com o intuito de prevenir a aparição de incapacidades em pacientes que já receberam alta.
\end{abstract}

Palavras-chaves: Hanseníase. Incapacidades. Prevalência.

\begin{abstract}
Introduction: Leprosy is a major public health concern. Brazil accounts for more than $90 \%$ of the cases in the Americas. Around 23\% of leprosy patients present some kind of disability after discharge. Methods: In the present study, the prevalence of disabilities among 169 patients (94 men and 75 women) with any of the clinical forms of leprosy, in the municipality of Várzea Grande, Mato Grosso, was estimated. They had already completed treatment over previous years. Interviews were conducted and clinical examinations of hand and foot sensitivity using Semmes-Weinstein filaments were carried out. Results: The prevalence of disabilities among the study sample was $42.6 \%$, among which $10.1 \%$ was grade 2 . A statistically significant relationship was found between disabilities and multibacillary forms of leprosy. Since the end of the treatment, sensitivity had worsened in 84 patients (50.2\%), and this was associated with presence of neural injury at the time of diagnosis. Conclusions: These results emphasize the importance of monitoring patients' neural functioning well beyond the time of concluding polychemotherapy, with the aim of preventing the appearance of disabilities among patients who have already been discharged.
\end{abstract}

Key-words: Leprosy. Disabilities. Prevalence.

1. Disciplina de Medicina Familiar e Comunitária, Faculdade de Ciências Médicas, Universidade de Cacoal. Cacoal, RO. 2. Departamento de Clínica Médica, Núcleo de Estudos de Doenças Infecciosas e Tropicais de Mato Grosso, Faculdade de Ciências Médicas, Universidade Federal de Mato Grosso. Cuiabá, MT.

Endereço para correspondência: Dr. José Maria Hernández Ramos. Disc Med Familiar e Comunitária FACIMED/Universidade de Cacoal. Av. Copacabana 559, Novo Cacoal, 78975-115 Cacoal, RO. Tel: 5565 9922-7702; 556999099995

e-mail: jogordo67@yahoo.com.br

Recebido para publicação em 02/10/2009

Aceito em 12/04/2010

\section{INTRODUÇÃO}

$\mathrm{Na}$ atualidade, existem aproximadamente 1.500.000 pacientes com hanseníase em todo o mundo, sendo que o Brasil contribui com cerca de $94 \%$ dos casos do continente americano e ocupa o segundo lugar em número absoluto de enfermos no ranking mundial, perdendo apenas para a Índia ${ }^{1,2}$.

Embora seja uma doença sem expressiva importância, no que tange à mortalidade, a real gravidade da hanseníase aparece quando consideramos o problema das incapacidades que produz. Cerca de $20 \%$ de todos os casos novos apresentam incapacidades no momento do diagnóstico e, outros 15\%, irão desenvolvê-las mesmo que todas as ações de saúde sejam realizadas adequadamente, seja no tratamento das neurites, dos estados reacionais e mesmo com a poliquimioterapia $(\mathrm{PQT})^{3,4}$.

No Brasil, aproximadamente $23 \%$ dos pacientes hansenianos apresentam algum tipo de incapacidade após a alta ${ }^{5,6}$. Mesmo assim, o Ministério da Saúde preconiza que, após a conclusão do tratamento adequado, ou seja poliquimioterapia por seis a doze meses, o paciente pode ser considerado como curado e retirado das estatísticas oficiais. Esses pacientes não são mais considerados como casos de hanseníase, mesmo que permaneçam com alguma sequela da doença ou apresentem episódios reacionais ${ }^{7}$.

O objetivo do presente trabalho foi investigar a situação relacionada à incapacidade dos pacientes com hanseníase oriundos do município de Várzea Grande, MT, após terem completado o esquema de PQT padronizado pelo Ministério da Saúde, com o intuito de identificar a evolução das sequelas ou o surgimento de novas incapacidades. 


\section{MÉTODOS}

Para análise das incapacidades, comparou-se a avaliação física no momento do estudo com aquela obtida no momento da alta terapêutica, nos pacientes notificados entre os anos 2004 e 2007, caracterizando uma coorte de pacientes com diferentes períodos de acompanhamento (coorte histórica aberta).

Os participantes foram submetidos a entrevista padronizada, sendo registradas as impressões do paciente sobre sua evolução e detalhes do seu tratamento. Informações complementares de cada paciente foram obtidas da ficha do SINAN e de prontuário médico das unidades de saúde onde foram tratados. Após a entrevista, foi realizado um exame clínico da sensibilidade em mãos e pés, mediante a utilização dos monofilamentos de Semmes-Weinstein aplicando o procedimento padrão ${ }^{8,9}$ e classificando a incapacidade física dos pacientes em três graus: grau 0 (sem incapacidade, não há comprometimento neural nem perda da sensibilidade), grau 1 (há incapacidade, mas sem deformidade) e grau 2 (alteração da sensibilidade superficial, com deformidade visível $)^{7}$. Foi considerado como tendo piora da sensibilidade o surgimento de alteração de sensibilidade em quem não a apresentava no momento da alta, assim como alteração em pelo menos dois pontos do território correspondente ao nervo estudado, em relação ao exame prévio.

\section{Análise estatística}

Para análise dos dados utilizou-se o pacote estatístico EpiData (versão 3.1). Foram calculadas as razões de odds ratio (OR) de piora das incapacidades ou de seu surgimento. Intervalos de confiança e o nível de significância foram estipulados em 95\%, e 5\% respectivamente. A seguir foram construídos modelos de regressão logística com o intuito de avaliar independência na associação com a presença e a evolução das incapacidades.

\section{Ética}

O estudo foi realizado após aprovação do anteprojeto pela Comissão de Ética em Pesquisa do Hospital Universitário Julio Muller, de Cuiabá, MT (protocolo No 403/CEP-HUJM/07). Os procedimentos estão em acordo com os padrões éticos da Declaração de Helsinki de 1964. Todos os pacientes foram informados dos objetivos da pesquisa, e solicitada autorização de cada um deles por meio de Termo de Consentimento Livre e Esclarecido.

\section{RESULTADOS}

A investigação foi realizada entre abril de 2007 e janeiro de 2009, em Várzea Grande, MT, selecionando-se pacientes a partir do arquivo do Centro de Doenças Tropicais, referência no município para acompanhamento de pacientes com hanseníase. Dos 757 pacientes tratados no período, foi possível contatar 598, por telefone. Desses, foram investigados 193 pacientes tratados ambulatorialmente, entre 2004 e 2008, sendo as perdas devidas mormente à mudança no número de telefone ou domicílio. Em 24 pacientes dos 193, não foi possível obter informações sobre o grau de incapacidade na época da alta nos registros médicos. As características da população não contatada eram similares às da população atingida, quanto à distribuição por idade e sexo. $\mathrm{Na}$ amostra selecionada, a forma indeterminada foi menos comum e a virchowiana foi a mais prevalente, quando comparadas com a população não contatada. Isto foi devido à centralização do atendimento do programa de hanseníase em centro de referência, determinando a agregação de maior número de casos graves, mormente virchowianos, determinando certa seleção da amostra. Somente dois casos de perda foram ocasionados por recusa de participação no estudo, não havendo vieses de autosseleção ou autoexclusão na amostra final.

Aidade média foi 48,3 (18 a 85) anos e houve predomínio do sexo masculino: 94 (55,6\%). Eram: 93 (55,4\%) de formas paucibacilares e 75 (44,5\%) de formas multibacilares. Houve predomínio das formas multibacilares no sexo masculino $(54,8 \%)$ e das paucibacilares no feminino (74,7\%; $\mathrm{p}=0,001)$. O intervalo médio entre o momento do diagnóstico e a entrevista foi de três anos, variando de menos de um ano a nove anos. O tempo médio de demora entre o diagnóstico e o início do tratamento foi de 10,8+/- 0,9 meses. A Tabela 1 resume algumas características quanto ao manejo dos pacientes, aparição de intercorrências e piora da sensibilidade.

Sobre o grau de incapacidade no momento da alta, $51(30,1 \%)$ apresentaram algum grau de incapacidade, sendo 34 (20,1\%) de grau

TABELA 1 - Dados evolutivos sobre a sensibilidade entre o término do tratamento e o momento da avaliação.

\begin{tabular}{|c|c|c|c|}
\hline & Número & Porcentagem ${ }^{*}$ & IC $95 \%$ \\
\hline \multicolumn{4}{|c|}{ Demora até o diagnóstico (meses) } \\
\hline$<6$ & 94 & 56,3 & $48,7-63,7$ \\
\hline $6-12$ & 34 & 20,6 & $14,8-27,0$ \\
\hline$>12$ & 39 & 23,4 & $17,4-30,2$ \\
\hline \multicolumn{4}{|c|}{ Educação em prevenção de incapacidades } \\
\hline $\operatorname{sim}$ & 40 & 23,7 & $17,7-30,5$ \\
\hline não & 58 & 34,3 & $27,5-41,7$ \\
\hline não procede ${ }^{* *}$ & 71 & 42,0 & $34,7-49,6$ \\
\hline \multicolumn{4}{|l|}{ Reações } \\
\hline $\operatorname{sim}$ & 67 & 39,9 & $32,7-47,4$ \\
\hline não & 101 & 60,1 & $52,6-67,3$ \\
\hline \multicolumn{4}{|l|}{ Acompanhamento } \\
\hline $\operatorname{sim}$ & 26 & 15,5 & $10,6-21,5$ \\
\hline não & 142 & 84,5 & $78,5-89,4$ \\
\hline \multicolumn{4}{|c|}{ Piora de sensibilidade } \\
\hline $\operatorname{sim}$ & 84 & 50,2 & $42,5-58,1$ \\
\hline não & 83 & 49,8 & $41,9-57,5$ \\
\hline
\end{tabular}

*a soma das categorias de cada variável analisada variou em consequência de não ter sido possível obter a informação de todos os casos.

** pacientes sem incapacidades no momento da alta. Pela legislação, sem indicação formal de orientação.

1 e 17 (10\%) de grau 2. Embora sem significância estatística $(p=0,2)$, houve maior prevalência de incapacidades entre os pacientes do sexo masculino (62,7\% vs 37,3\%; Tabela 2). Quando analisados apenas os pacientes com incapacidade de grau 2 , revelou-se diferença estatística entre os sexos ( $14,9 \%$ vs $4 \%$, $\mathrm{p}=0,03)$.

No exame físico atual, dos 169 pacientes, 85 (50,3\%) apresentaram algum grau de incapacidade, sendo 66 (39\%) de grau 1 e $19(11,3 \%)$ de grau 2 (Tabela 3). Houve maior prevalência de incapacidades entre os pacientes com formas multibacilares de hanseníase em comparação às formas paucibacilares (Tabela 3 ). Essa associação manteve-se estatisticamente significante mesmo quando ajustada para gênero e faixa etária em análise multivariada por regressão logística tanto no momento da alta $(\mathrm{OR}=2,3$; 
TABELA 2 - Grau de incapacidade no momento de receber alta, no momento do estudo, e alteração da sensibilidade na amostra por distribuição de gêneros*.

\begin{tabular}{|c|c|c|c|c|c|c|c|c|}
\hline & \multirow[b]{2}{*}{$\mathrm{n}^{\circ}$} & \multirow[b]{2}{*}{$\%$} & \multirow[b]{2}{*}{ IC $95 \%$} & \multicolumn{2}{|c|}{ Masculino } & \multicolumn{2}{|c|}{ Feminino } & \multirow[b]{2}{*}{$\mathrm{p}$} \\
\hline & & & & $\mathrm{n}^{\mathrm{o}}$ & $\%$ & $\mathrm{n}^{\mathrm{o}}$ & $\%$ & \\
\hline \multicolumn{9}{|c|}{ Incapacidade na alta } \\
\hline grau 0 & 118 & 69,9 & $63,1-76,7$ & 62 & 65,9 & 56 & 74,7 & - \\
\hline grau 1 & 34 & 20,1 & $14,1-26,1$ & 18 & 19,2 & 16 & 21,3 & $0,8^{* *}$ \\
\hline grau 2 & 17 & 10,0 & $5,4-14,6$ & 14 & 14,9 & 3 & 4,0 & $0,03^{* *}$ \\
\hline \multicolumn{9}{|c|}{ Incapacidade no estudo } \\
\hline grau 0 & 97 & 57,4 & $49,8-64,7$ & 47 & 50 & 50 & 66,7 & \\
\hline grau 1 & 55 & 32,5 & $25,8-39,9$ & 35 & 37,2 & 20 & 26,7 & $0,1^{* *}$ \\
\hline grau 2 & 17 & 10,1 & $6,2-15-3$ & 12 & 12,8 & 5 & 6,7 & $0,1^{* *}$ \\
\hline \multicolumn{9}{|c|}{ Piora sensibilidade } \\
\hline $\operatorname{sim}$ & 84 & 50,2 & $42,5-58-1$ & 52 & 55,9 & 32 & 43,2 & \\
\hline não & 83 & 49,8 & $41,9-57,5$ & 41 & 42,1 & 42 & 56,8 & 0,07 \\
\hline
\end{tabular}

${ }^{*}$ A soma das categorias de cada variável analisada variou em consequência de não ter sido possível obter a informação de todos os casos. ${ }^{* *}$ Comparando a primeira categoria com as restantes, aplicado teste exato de Fisher.

IC $\left.95 \%=1,5-3,5 ; \mathrm{p}=0,001^{\mathbf{0}}\right)$, quanto no avaliação atual $(\mathrm{OR}=3,1$; IC $95 \%=1,5-6,3 ; \mathrm{p}=0,002^{\mathbf{0}}$ ).

Piora da sensibilidade foi mais frequente nos pacientes que referiram ser trabalhadores braçais, nos que já apresentavam lesão neural e naqueles com incapacidade grau 2 no momento da alta (Tabela 4). A forma clínica da hanseníase não influenciou. Dentre 42 pacientes, que receberam orientações para prevenir o surgimento
TABELA 3 - Grau de incapacidade ( 0 = ausência de incapacidade, 1 = leve, 2 = grave) por forma clínica em 169 pacientes com hanseníase no momento da alta e no momento atual*.

\begin{tabular}{|c|c|c|c|c|c|c|c|c|c|c|c|}
\hline \multirow[b]{4}{*}{ Forma clínica } & \multicolumn{11}{|c|}{ Grau de incapacidade } \\
\hline & \multicolumn{6}{|c|}{ no momento de alta } & \multicolumn{5}{|c|}{ no momento do estudo } \\
\hline & \multicolumn{2}{|r|}{0} & \multicolumn{2}{|r|}{1} & \multicolumn{2}{|c|}{2} & \multicolumn{2}{|c|}{0} & \multicolumn{2}{|r|}{1} & 2 \\
\hline & $\mathrm{n}^{\mathrm{o}}$ & $\%$ & $\mathrm{n}^{\mathrm{o}}$ & $\%$ & $\mathrm{n}^{\mathrm{o}}$ & $\%$ & $\mathrm{n}^{\mathrm{o}}$ & $\%$ & $\mathrm{n}^{\mathrm{o}}$ & $\%$ & $\mathrm{n}^{\circ} \quad \%$ \\
\hline Paucibacilar & 82 & 83,7 & 10 & 10,2 & 6 & 6,1 & 74 & 68,5 & 28 & 25,9 & 65,0 \\
\hline Multibacilar & 36 & 70,2 & 24 & 20,2 & 10 & 9,5 & 33 & 55,7 & 38 & 34,4 & 139,9 \\
\hline
\end{tabular}

${ }^{*}$ A soma das categorias de cada variável analisada variou em conseqüência de não ter sido possível obter a informação de todos os casos.

de incapacidades, $24(57,1 \%)$ apresentaram piora da sensibilidade. Nos 60 pacientes que não receberam nenhuma orientação, $38(63,3 \%)$ pioraram. Em 72 pacientes, não foi indicada orientação para prevenção de incapacidades, pela forma clínica ou pelo resultado satisfatório do tratamento sem intercorrências. Dentre esses pacientes, 28 (38,8\%) apresentaram piora da sensibilidade. $\mathrm{Na}$ análise multivariada, controlada por gênero e faixa etária, a piora da sensibilidade continuou associada à presença de lesão neural no momento da alta $(\mathrm{OR}=2,7$; IC95\%=1,4-5,3; $\mathrm{p}=0,003^{\mathbf{2}}$ ). Dentre os 169 casos em que foi possível a comparação de grau de incapacidade no momento da alta com o momento atual, encontrou-se que de 118 pacientes que finalizaram o tratamento sem incapacidade, $31(26,2 \%)$ evoluíram para o grau 1. Dentre os 34 pacientes que apresentavam grau 1 de incapacidade, dois $(5,8 \%)$ pioraram e apresentaram grau 2 .

TABELA 4 - Comparação da frequência de fatores de risco nos grupos com piora ou não da sensibilidade em pacientes que receberam tratamento por hanseníase em centros de saúde de Várzea Grande, MT, de 2004 a 2007.

\begin{tabular}{|c|c|c|c|c|c|c|c|c|}
\hline & \multirow{2}{*}{\multicolumn{2}{|c|}{ Piora }} & \multirow{2}{*}{\multicolumn{2}{|c|}{ Não-piora }} & \multirow[b]{3}{*}{ OR (IC 95\%) } & \multirow[b]{3}{*}{$\mathrm{P}$} & \multirow{3}{*}{$\begin{array}{c}\text { Análise } \\
\text { multivariada }^{a} \\
\text { OR (IC 95\%) }\end{array}$} & \multirow{3}{*}{$\begin{array}{l}\text { P Análise } \\
\text { multivariada }\end{array}$} \\
\hline & & & & & & & & \\
\hline & $\mathrm{n}^{\mathrm{o}}$ & $\%^{*}$ & $\mathrm{n}^{\mathrm{o}}$ & $\%^{*}$ & & & & \\
\hline trabalho braçal & 3 & 53,4 & 2 & 34,6 & $2,2(1,04-4,6)$ & 0,03 & & \\
\hline trabalho não braçal & 27 & 46,6 & 49 & 65,4 & & & & \\
\hline \multicolumn{9}{|c|}{ Tempo demora $(\text { anos })^{* * *}$} \\
\hline $0-1$ & 15 & 16,6 & 27 & 32,1 & $1,0^{+}$ & - & & \\
\hline $2-5$ & 65 & 72,2 & 50 & 59,5 & $2,3^{+}(1,06-5,2)$ & 0,02 & & \\
\hline$>5$ & 10 & 11,2 & 7 & 8,4 & $2,5^{+}(0,7-9,5)$ & 0,1 & & \\
\hline \multicolumn{9}{|c|}{ Lesão neural presente no diagnóstico } \\
\hline não & 45 & 50,5 & 61 & 74,3 & & & & \\
\hline $\operatorname{sim}$ & 44 & 49,4 & 21 & 25,6 & $2,8(1,4-5,7)$ & 0,001 & $2,7(1,3-5,8)$ & 0,007 \\
\hline $\operatorname{sim}$ & 24 & 57,1 & 18 & 42,9 & $1,3(0,5-3,1)$ & 0,5 & & \\
\hline não & 38 & 63,3 & 22 & 36,7 & & & & \\
\hline Sem indicação ${ }^{* * * *}$ & 28 & 38,9 & 44 & 61,1 & & & & \\
\hline \multicolumn{9}{|c|}{ Grau de incapacidade na alta } \\
\hline 0 & 51 & 60,7 & 66 & 79,5 & $1,0^{+}$ & - & 1,0 & - \\
\hline 1 & 21 & 25,0 & 13 & 15,6 & $2,0^{+}(0,9-4,9)$ & 0,06 & $1,3(0,5-3,1)$ & 0,5 \\
\hline 2 & 12 & 14,3 & 4 & 4,8 & $\left.3,9^{+} 1,0-15,2\right)$ & 0,04 & $1,8(0,5-6,7)$ & 0,3 \\
\hline
\end{tabular}

*A soma das categorias de cada variável analisada variou em consequência de não ter sido possível obter a informação de todos os casos.

${ }^{* *}$ Excluídos os pacientes que não trabalhavam no momento do estudo. Variável não incluída na análise multivariada pela perda de informação (vide texto).

***Transcorrido desde o momento do diagnóstico até o momento do estudo.

****Sem indicação de educação para prevenir incapacidades.

${ }^{\dagger}$ Oddis ratio calculados tendo como base a primeira categoria.

ancluindo as variáveis com relação estatisticamente significante na análise univariada, exceto a variável "atividades laborais”.

(1) ambos modelos com 168 observações, 2 modelo com 169 observações. 


\section{DIsCUSSÃo}

A prevalência de incapacidade de grau 2 (10\%) no momento da alta encontrada no presente estudo pode ser considerada média, segundo os indicadores epidemiológicos propostos pelo Ministério da Saúde ${ }^{10}$. Este resultado difere dos achados de Deepak ${ }^{11}$, que encontrou alta prevalência de incapacidade grau 2 em levantamentos internacionais, podendo variar de $17 \%$ a $50 \%$ após a alta. Se considerarmos a presença de qualquer alteração da sensibilidade (graus 1 e 2), a prevalência total alcançou $30,1 \%$. Existem diferentes estudos sobre a prevalência de incapacidades no Brasil ${ }^{12-14}$. Invariavelmente, a prevalência de pacientes com alguma incapacidade física se manteve entre 20\% a $30 \%$. Segundo o Ministério da Saúde, 23\% dos pacientes com hanseníase apresentam algum grau de incapacidade após alta, e no período de 1987 a 1996, encontrou-se que, do total de casos novos notificados, $16 \%$ já apresentavam grau 1 de incapacidade, sendo a proporção de casos com grau 2 de $8 \%{ }^{15}$. Portanto, os resultados do presente estudo se situam no nível de prevalência esperada.

Os resultados obtidos também estão de acordo com a informação de que cerca de $20 \%$ dos pacientes com hanseníase, podem apresentar incapacidades físicas, durante ou depois do tratamento, chegando a necessitar de algum tipo de continuidade dos cuidados médicos ${ }^{16}$. No Brasil, a grande maioria dos pacientes não apresenta incapacidades no início da doença ${ }^{17,18}$. De modo que os pacientes com algum grau de incapacidade, podem significar detecção tardia da doença. Neste trabalho, a elevada percentagem de retardo no diagnóstico superior a seis meses (44\%) e, sobretudo, superior a 12 meses (23,4\%) chama a atenção para a existência de falhas no programa para detecção precoce dos doentes.

No presente estudo, a única variável relacionada com a presença de incapacidades, tanto no momento do diagnóstico, quanto no momento da realização do estudo foi a forma multibacilar da hanseníase. A frequência de quadros reacionais esteve dentro do previsto, segundo os dados da literatura ${ }^{8}$. Embora a presença de reações possa condicionar piora neurológica, o adequado manejo das mesmas é o que irá, de fato, determinar a evolução dos pacientes. A ausência de diagnóstico precoce das neuropatias, o receio e a não adoção de protocolos no uso da prednisona são achados frequentes, que determinam, muitas vezes, as deficiências e incapacidades ${ }^{19,20}$. A inexistência de dados nos prontuários consultados não permitiu estudar a relação entre os diferentes tratamentos dos quadros reacionais e aparição de piora na sensibilidade.

Só a quarta parte dos pacientes recebeu orientações para a prevenção de incapacidades . A falta de conhecimentos sobre estas técnicas pela grande maioria dos pacientes pode ter condicionado o desenvolvimento de lesões em alguns casos. Acredita-se que uma avaliação neurológica abrangente deveria ser repetida duas ou três vezes por ano e até por cinco anos após o término do tratamento farmacológico ${ }^{3}$. Portanto, segundo os dados da presente amostra, existe uma falha importante no esquema de acompanhamento dos pacientes após o diagnóstico. A análise estatística não mostrou associação $(\mathrm{p}=0,4)$, provavelmente pelo tamanho da amostra.

Mais da metade dos pacientes apresentou piora da sensibilidade de mãos e pés. Segundo De Oliveira e cols ${ }^{16}$, dentre os pacientes que não apresentam incapacidades no final do tratamento, $5 \%$ dos paucibacilares e até $20 \%$ dos multibacilares desenvolvem, posteriormente, alguma alteração da sensibilidade.
O maior tempo transcorrido desde o momento do diagnóstico até o estudo não foi relacionado com piora na sensibilidade. Os pacientes que apresentavam algum tipo de comprometimento neural no momento do diagnóstico tiveram maior risco de piora da sensibilidade após finalizar o tratamento. Nestes pacientes, sem acompanhamento adequado, a lesão neural pode acontecer de maneira silenciosa (sem dor) e evoluir com piora da função, muitas vezes sem o próprio paciente perceber ${ }^{3,4,16}$. É interessante destacar o elevado número de pacientes que finalizaram o tratamento sem alterações da sensibilidade e que, transcorridos vários meses, apresentaram leve a moderada hipoestesia, perdendo a sensibilidade protetora e entrando no grupo de risco para

desenvolvimento de lesões. $\mathrm{O}$ fato de ter recebido orientações para prevenção de incapacidades no domicílio não constituiu um fator de proteção contra incapacidades na amostra analisada, pois em ambos os grupos de pacientes, orientados e não orientados, a piora de sensibilidade foi semelhante. Além disso, as altas taxas de alterações da sensibilidade, entre os pacientes nos quais teoricamente não era necessário nenhum tipo de intervenção educativa, indica a necessidade de monitorar atentamente a função neural destes pacientes depois de finalizado o tratamento, mesmo após alta por cura.

Em suma, a prevalência de incapacidades nesta amostra de hansenianos, que receberam alta nos quatro anos que antecederam o estudo foi de $42,6 \%$, com $10,1 \%$ apresentando grau 2 . Houve associação entre a presença de incapacidades com as formas multibacilares da hanseníase. Houve também associação entre a piora da sensibilidade e a presença de lesão neural já no momento do diagnóstico. $\mathrm{O}$ fato de metade dos pacientes ter desenvolvido progressão de déficit de sensibilidade indica a necessidade de monitorar a função neural dos hansenianos além do momento de finalizar a poliquimioterapia, para evitar o surgimento de incapacidades em pacientes que já tiveram alta.

\section{CONFLITO DE INTERESSE}

Os autores declaram não haver nenhum tipo de conflito de interesse no desenvolvimento do estudo.

\section{REFERÊNCIAS}

1. Organização Panamericana da Saúde. Monitoramento da Eliminação da Hanseníase (LEM). Brasília: OPAS; 2004. OPS/DPC/CD 288/4 (11MB).

2. Noto S, Nunzi E. Global and regional annual new case detection of leprosy reported by de World Health Organization. Lepr Rev 2008; 79: 1-5.

3. Duerksen F. Reabilitação. In: Opromolla DVA, editor. Noções de Hansenologia. Bauru: Centro de Estudos Dr.Reynaldo Quagliato, Instituto Lauro de Souza Lima; 2000. p.113-115.

4. Duerksen F. Reabilitação. In: Opromolla DVA, Baccarelli R, editores. Prevenção de incapacidades e reabilitação em hanseniase. Bauru, Instituto Lauro Souza Lima; 2003. p. 3-4.

5. Gonçalves SD, Sampaio RF, Antunes CMF. Fatores preditivos de incapacidades em pacientes com hanseníase. Rev Saude Publica 2009; 43: 267-274.

6. Helene LMF, Leão VM, Minakawa MM. The social situation and the present of the physical disabilities among leprosy patients registered at a public health center in São Paulo city. Hansenol Int 2001; 26: 5-13.

7. Ministério da Saúde. Secretaria de Políticas de Saúde. Departamento de Atenção Básica. Área Técnica de Dermatologia Sanitária. Hanseníase: atividades de controle e manual de procedimentos. Brasília; 2001. 
8. Secretaria do Estado de Saúde de Minas Gerais. Superintendência de Atenção à Saúde. Diretoria de Normalização e Atenção à Saúde. Coordenadoria Estadual de Dermatologia Sanitária de Minas Gerais. Como reconhecer e tratar as reações hansênicas; 2005.

9. Souza A, Nery CAS, Marciano LHS, Garbino JA. Avaliação da neuropatia periférica diabética: correlação entre a sensibilidade cutânea dos pés, achados clínicos e eletroneurográficos. Act Fisiatr 2005; 12: 87-93.

10. Ministério da Saúde. Fundação Nacional de Saúde. Guia de Vigilância Epidemiológica Vol I. Hanseníase; 2002.

11. Deepak S. Answering the rehabilitation needs of leprosy affected persons in integrated setting through primary health care services and community based rehabilitation. Indian L Lepr 2003; 75: 127-142.

12. Rosa RS. Levantamento de incapacidades em pacientes hansenianos do município de Catanduva-SP, 1986: condições de atendimento para sua prevenção nos treze municípios abrangidos pelo Escritório Regional de Saúde de Catanduva ERSA 30. Tese apresentada à Universidade de São Paulo, Faculdade de Medicina de Riberão Preto, para obtenção do grau de Mestre, Ribeirão Preto; 1987.

13. Goulart IB, Dias CM, Oliveira ACS, Silva AA, Alves RR, Quaresemin CR, et al Grau de incapacidade: indicador de prevalência oculta e qualidade do programa de controle de hanseníase em um centro de saúde-escola no município de Uberlândia. Hansen Int 2002; 27: 5-13.

14. Raposo MT, Raposo AV, Medeiros JLA. Distribuição territorial das incapacidades físicas determinadas pela hanseníase no município de Campina Grande-PB. Hansen Int 2005; 30: 119.

15. Ministério da Saúde. Secretaria de Políticas de Saúde. Departamento de Gestão de Políticas Estratégicas. Relatório de atividades da área técnica de dermatologia sanitária. Brasília; 1999.

16. De Oliveira CR, De Alencar MJ, De Sena NSA, Lehman LF, Schereuder PA. Impairments and Hansen's disease control in Rondônia state, Amazon region of Brazil. Lepr Rev 2003; 74: 337-348.

17. Schiapati T. Grau de incapacidade atribuído aos casos novos em hanseníase no momento de sua admissão. Hansenol Int 2005; 30: 124

18. Suárez REG, Lombardi C. Estimado de prevalência de lepra. Hansenol Int 1997 22: $31-35$

19. Selvaraj G, Prabakar N, Muliyil J, Martin G. Incidence of disabilities among multi-bacillary cases after initiation of multidrug therapy and factors associated with de risk of developing disabilities. Indian J Lepr 1998; 70(supl): 11-16.

20. Sharma P, Kar HK, Beena KR, Kaur H, Narayan R. Disabilities in multibacillary leprosy patients: defore, during and after multidrug therapy. Indian J Lepr 1996; 68: 127-136. 